\title{
SoRoute: a reliable and effective social-based routing in cognitive radio ad hoc networks
}

\author{
Tao Jing ${ }^{\text {* }}$, Jie Zhou ${ }^{1}$, Hang Liu², Zhewei Zhang ${ }^{1}$ and Yan Huo ${ }^{1}$
}

\begin{abstract}
A cognitive radio ad hoc network (CRAHN) can be considered as a special delay tolerant network (DTN) that is composed of mobile secondary users (SUs) with social characteristics. Given intermittent connectivity and spectrum availability, it is a challenging issue regarding how to transmit messages between SUs in a reliable and effective way in CRAHNs. To tackle this challenge, we propose a social-aware opportunistic routing and relay selection scheme, called SoRoute, which first predicts the link reliability based on a new social-relationship-aware mobility model and then fuses the relationships of SUs to make a routing and relay decision. In our design, different prediction schemes are employed for the nodes with different relationships. A message is forwarded to the relay node with the largest encounter probability with the destination. The evaluation results demonstrate that our social-based opportunistic routing scheme significantly improves the performance compared to the existing routing schemes such as Direct Delivery, First Contact, MaxProp, and Prophet.
\end{abstract}

Keywords: Cognitive radio ad hoc networks; Delay tolerant networks; Social relationship; Routing; The ONE simulator

\section{Introduction}

A cognitive radio (CR) [1] is a promising technology to address the spectrum scarcity problem and improve spectrum utilization, which enables secondary users (SUs) opportunistically access the vacant spectrum bands allocated to primary users (PUs) [2-8]. Due to the specific characteristics of $\mathrm{CR}$, it has broad application prospects. For example, CR technology can be combined with mobile ad hoc networks, which is called cognitive radio ad hoc networks (CRAHNs) [9], in which wireless devices can dynamically establish networks using the vacant spectrum bands allocated to PUs without the need of fixed infrastructures. However, the distinct characteristics of CRAHNs, such as the mobility of nodes and the limitation of spectrum, result in intermittent connectivity in such networks. The lack of a sustainable end-to-end path makes CRAHNs appear to be delay tolerant networks (DTNs) [10,11]. All of the DTN routing methods share a similar characteristic, the 'store-carry-forward' fashion. That means a DTN node can store and carry the data until it encounters a suitable node.

\footnotetext{
*Correspondence: tjing@bjtu.edu.cn

1 School of Electronics and Information Engineering, Beijing Jiaotong

University, 100044 Beijing, China

Full list of author information is available at the end of the article
}

From a routing perspective, a link is considered to be reliable if the link duration time is longer than the time required for transmitting data. It is an unreliable link otherwise. An unreliable link may be broken frequently. When any link of a path fails, this path has to be either repaired by finding another link or replaced entirely with a new path. These rerouting operations waste the scarce radio resource and battery power, and rerouting delay may also affect quality of service (QoS) and degrade network performances [12]. As a result, the reliability of a link is an important criterion of measuring whether a link can be used to forward a message. Furthermore, due to the limitation of spectrum availability and node mobility, routing in CRAHNs may incur a long delay. Thus, it is a challenging problem how to transmit messages reliably and efficiently in CRAHNs.

Most of the existing DTN routing schemes [13-17] are based on classic ad hoc networks, ignoring the spectrum availability. However, routing in CRAHNs should take into consideration not only the mobility of nodes but also the spectrum availability which is under the condition that SUs can not generate interference to PUs. In addition, many previous works on routing in ad hoc networks assumed that messages would be forwarded completely once two nodes encounter with each other [18].

\section{Springer}

(c) 2014 Jing et al: licensee Springer. This is an Open Access article distributed under the terms of the Creative Commons Attribution License (http://creativecommons.org/licenses/by/4.0), which permits unrestricted use, distribution, and reproduction in any medium, provided the original work is properly credited. 
Obviously, this assumption is unreasonable when message size is large and mobility is high. Based on the analysis above, a link reliability prediction scheme is necessary in CRAHNs. Mobile SUs have social relationships. Portable devices, such as smart phones or ipads, are always carried by human beings. Therefore, the mobility pattern of these nodes corresponds to that of their users. Empirical observations indicate there exists certain social relationships between different people, and people of different relationships have different social behaviors. For example, friends stay together longer than strangers, and colleagues or classmates meet more frequently than distant relations. A link reliability can be better predicted and a routing decision can be better made by exploring these social relationships. A few of existing works considers the concept of social networks in DTN routing. However, most of these studies are made from the view of social characteristics such as community and centrality. Few of them model and conduct qualitative analysis on the link duration and encounter probabilities of SUs based on the different relationships of the nodes.

In this paper, we propose a novel opportunistic routing and relay selection scheme, called SoRoute, which forwards the messages based on the social relationships between the nodes in a reliable and efficient way. The main contributions of this paper are summarized as follows:

- A new mobility model is proposed, which can represent the mobility patterns of nodes with the real-world social relationships.

- Based on this model, a link reliability metric and a prediction algorithm are derived for the SUs with different relationships, taking into account SUs' transmission range limitation and interference with PUs.

- We propose a routing and relay selection protocol based on the social-aware link reliability.

- We demonstrate the proposed social-based opportunistic routing scheme that significantly improves the performance compared to the existing routing schemes such as Direct Delivery, First Contact, MaxProp, and Prophet.

To the best of our knowledge, this is the first work to combine the concept of social relationships with the prediction of link reliability for routing and relay selection. The rest of this paper is organized as follows. Section 2 presents the related work. In section 3, network model and mobility model are presented. Section 4 gives a brief introduction of the social relationships between human beings. The link reliability prediction algorithm is given in section 5 . The social-based opportunistic routing scheme is described in section 6 . In section 7 , we demonstrate the validity of our link reliability prediction algorithm. The performance of the proposed routing scheme is also evaluated. Section 8 gives the conclusions.

\section{Related work}

The existing DTN routing schemes can be categorized into two classes, flooding $[13,19,20]$ and forwarding [21-23]. A flooding approach is based on the principle of forwarding messages to all the neighboring nodes. Due to the limitation of bandwidth and buffer space, the flooding approach performs poorly in many situations. A forwarding strategy uses certain knowledge about the network to select a best path to the destination. Cheng et al. [21] propose a location-based routing method for DTN which can estimate the minimum distance between the source and destination, then messages are transmitted through the best path. In [22], the weight is assigned to each node, which represents the suitability of the node to deliver messages to a given destination. This algorithm only delivers messages to the relay which has a better weight than the current node, thus it is called gradient routing strategy. Srinivasa and Krishnamurthy [23] use 'conditional inter-meeting time' as a metric to estimate the best path towards the destination, which can achieve a high delivery rate and a short end-to-end delay.

In addition, social-based routing algorithms have been proposed to exploit certain social characteristics in DTN [14-17,24,25]. Hui et al. [14] propose a forwarding strategy named Bubble Rap, which focuses on the community and centrality of the nodes. It delivers messages by using the global ranking and the local ranking. In [15], a SimBet Routing is proposed which exploits the exchange of pre-estimated betweenness centrality metrics and locally determined social similarity to the destination node. Wang et al. [16] exploit the spatial locality, spatial regularity, and activity heterogeneity of human mobility to select relays. Wang et al. [17] summarize the behavioral traits of human beings employed by existing PSN routing schemes and give a survey on PSN routing techniques. Bulut and Szymanski [24] introduce a new metric for detecting the quality of friendships, and then the temporally differentiated friendships are used to make the forwarding decisions of messages. Gao et al. [25] study multicast in DTNs from the social network perspective, in which relay selections for multicast is formulated as a unified knapsack problem by exploiting node centrality and social community structures.

However, none of the above works considered CRAHNs. In CRNs, SUs can opportunistically access the vacant bands allocated to PUs [2-8]. Huang et al. [11] study a social-based routing in intermittently connected mobile cognitive radio network, but it did not consider the link reliability in message forwarding. Therefore, a 
link duration prediction scheme is needed to analyze the reliability of a link. The existing work on link reliability prediction is limited [26-28]. Han et al. [26] proposed an algorithm, named LBRP, which can predict link availability over a short time by a rough estimation of the distance between two nodes. However, LBRP is based on the random walk mobility model, which is too simple to reflect the mobility in real world. In $[27,28]$, a prediction-based link reliability estimation algorithm and its enhanced version were developed. These two algorithms try to predict the probability of an active link between two nodes being continuously available for a certain period of time. However, they are based on statistics and can only represent the general tendency of the link availability. In addition, a link prediction scheme is proposed for CR-MANETs [29], which is aware of the interference to primary users. However, this link prediction scheme is only based on the current node movement and cannot accurately calculate the link reliability. To the best of our knowledge, there is no link reliability prediction scheme available in literature that takes into account the social characteristics and mobility of nodes, as well as interference to the PUs.

\section{System model}

\subsection{Network model}

We consider a fixed primary network and a CR mobile ad hoc secondary network as shown in Figure 1. The primary network consists of a primary base station (BS) and $M$ PUs with known locations, denoted as $\left\{\mathrm{PU}_{1}, \mathrm{PU}_{2}, \cdots\right.$, $\left.\mathrm{PU}_{M}\right\}$. Coexisting with this primary network is a mobile ad hoc secondary network, which is composed of $N$ SUs (i.e., mobile devices), denoted by $\left\{\mathrm{SU}_{1}, \mathrm{SU}_{2}, \cdots, \mathrm{SU}_{N}\right\}$.
The primary spectrum band contains $L$ orthogonal subchannels, and each PU is allocated a specific sub-channel, simply assuming sub-channel $k$ for $\mathrm{PU}_{k}$. PUs have the rights of accessing the licensed bands for communications with the primary BS, while SUs can only opportunistically access the sub-channels for ad hoc device-to-device transmissions. In this model, we adopt an underlay spectrum access mode, in which SUs can access the sub-channels only when the interference temperature of PUs is lower than the threshold that PUs can tolerate. Since SINR is a function of transmit power and distance, for simplicity, we assume that a SU transmits with a fixed low power. The interference range of $\mathrm{SU}_{i}, \rho_{S i}$, is defined as the distance between $\mathrm{SU}_{i}$ and node $r$, out of which the interference of $\mathrm{SU}_{i}$ 's transmission to the signal reception of node $r$ can be ignored. In addition, PUs may have interference to SUs. The interference range of $\mathrm{PU}_{k}, \rho_{P k}$, is thus defined in a similar way. A definition for $\mathrm{SU}_{i}$ detecting channel $k$ available is given as follows:

Definition 1. (Channel availability) $S U_{i}$ detects channel $k$ is available when it satisfies: (1) $S U_{i}$ is out of the interference range $\rho_{P k}$ of $P U_{k}$; (2) $S U_{i}$ is out of the interference range $\rho_{P_{b s}}$ of PU base station; and (3) $P U_{k}$ and $P U$ base station are both out of the interference range $\rho_{S i}$ of $S U_{i}$.

In this model, we assume that SUs are mobile devices with $\mathrm{CR}$ functionality, which can detect the available channel through spectrum sensing. One condition for a successful communication between two SUs is that there exists at least one common available channel between them. In other words, only when two SUs detect the same available channel can they transmit messages on this

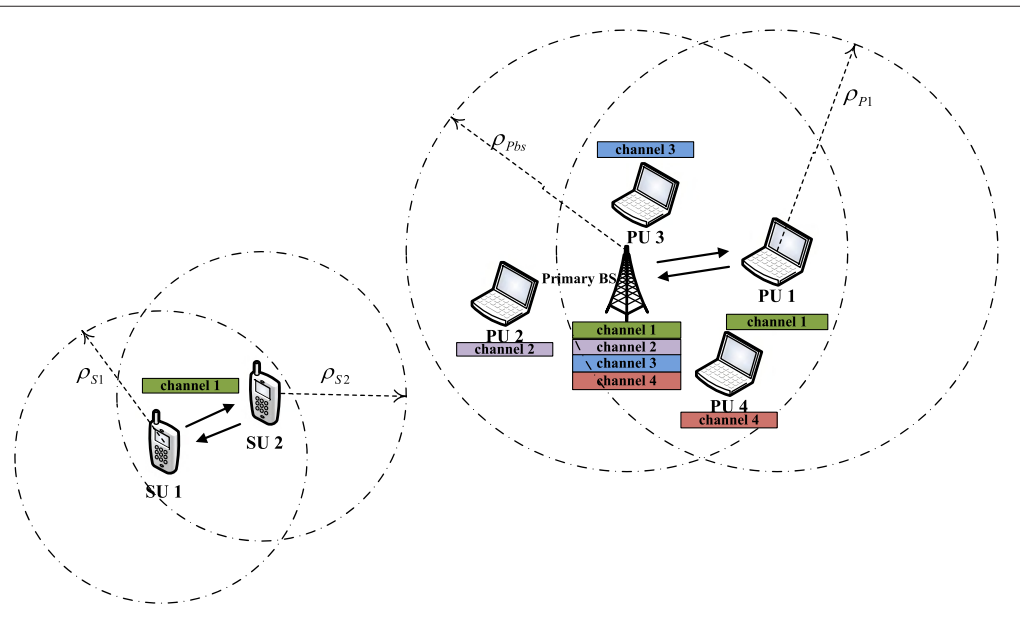

Figure 1 Network model. The laptops are denoted as primary users, and the cell phones represent secondary users. The dotted circles denote the interference range of PUs or SUs. In this example, there is a primary network with one PU base station and four primary users as well as a cognitive radio ad hoc network. Each PU is allocated a specific sub-channel. According to Definition 1, both $\mathrm{SU}_{1}$ and $\mathrm{SU}_{2}$ detect that channel 1 is available, therefore channel 1 is a common channel for their message transmission. 
common channel. The other condition is that the two SUs are within their transmission range. Due to the limitation of the transmission power, the distance between two SUs can not be far away. Here, we make an assumption that only when the distance between two nodes is no larger than $R_{0}$ can they communicate successfully, where $R_{0}$ is the transmission distance of each SU.

\subsection{Mobility model}

As it is mentioned above, PUs in this model are static nodes, while SUs are mobile devices, such as smart phones or ipads that are carried by human beings. As a result, the mobility pattern of SUs corresponds to that of their users. Empirical observation says the mobility of human beings has the characteristic of both randomness and sociality. Randomness means people can change their moving speed and direction arbitrarily, while sociality signifies that there exists certain regulations during the movement of each node. The traditional random walk mobility model is then no longer suitable. A new mobility model that can reflect the sociality between nodes is quite essential. In this paper, we first propose a social-based mobility model to simulate the mobility pattern of SUs. In this model, SUs are assumed to have their position information available, which could be obtained via techniques proposed in $[30,31]$. The whole area is partitioned into multiple zones $\left(Z_{m}, m=1,2, \cdots, \omega\right)$, which satisfies $\cup Z_{m}=\Omega$ and $\cap Z_{m}=\emptyset$. Each zone is determined by its functions, such as teaching buildings, dormitory areas, playgrounds, and dining rooms in a university campus. For different nodes, the probability of entering each zone is different. Let $\chi_{i}^{m}$ denote the probability for node $i$ moving towards zone $m$. We assign a special landmark to each zone to represent all the places in this zone. For simplicity, the landmark is located at the center of the zone, and it is expressed as $C_{m}$ for zone $Z_{m}$. Each node's movement can be divided into a sequence of time intervals called mobility epochs $\xi$, during which a node moves in a constant direction and speed [26]. The epoch length is constant for all the nodes. The speed of each SU node in one epoch is uniformly distributed over $\left(v_{\min }, v_{\max }\right)$, while the direction is discretely distributed, which points to $Z_{m}$ with probability $\chi_{i}^{m}$ for node $i$. After each epoch ends, the nodes will make a decision whether to have a pause. If there is any friend around him, the node and his friends will have a short stay. The pause time obeys a power law distribution because few people stay together for a long time, and most have a short stay. Speed, direction, epoch length, and pause time are uncorrelated [26]. For a zone $Z_{m}$, the more frequently a node visits, the larger the probability of moving towards $C_{m}$ is. The socially closer the two nodes are, the longer time they stay together. Obviously, the new mobility model is more coincidental to the mobility pattern of human beings in the real world.

\section{Analysis of social relationships}

The social relationships among mobile nodes are more likely to be long-term characteristic and less volatile than node mobility. People with different relationships may have different encounter frequencies and encounter durations. Therefore, the social relationship can be regarded as an important criterion to predict the encounter duration between two users, and it can also be used as a metric to guide the relay selection.

\subsection{Classification of social relationships}

According to Granovetter's theory [32], the social relationship between people can be divided into four categories according to their encounter frequencies and encounter durations, as shown in Figure 2. The $x$-axis denotes the encounter duration, and the $y$-axis denotes the encounter frequency.

I. Close friends: Nodes in this relationship have not only high encounter frequencies but also long durations just like close friends in the real world. Of course, this relationship is not common in the real world.

II. Familiar strangers: Two nodes are defined as familiar strangers if they encounter frequently but the duration of each meeting is short. For example, students who study in the same teaching building but not knowing each other encounter with each other several times a day; however, there exists no communication when they meet. As a result, their encounter duration is short.

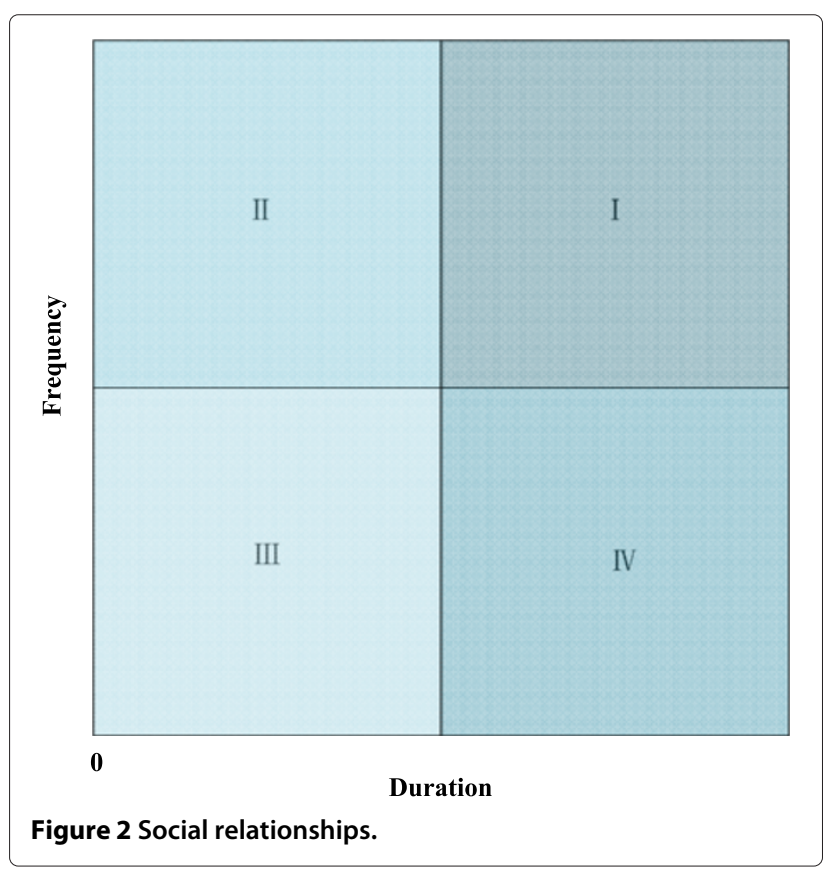


III. Strangers: Nodes who have this relationship have both a low encounter frequency and a short duration time, just as the strangers in the real world, and this relationship is quite common.

IV. Ordinary friends: If the encounter duration of two nodes is quite long once they meet each other, even if the encounter frequency is not high, we call these nodes ordinary friends. Just like we can not meet our friends frequently, but once we meet with each other, we will stop or walk together to have a conversation.

\subsection{Application of social relationships in CRAHNs}

From the analysis above, we can get that any two CR nodes in CRAHNs have a certain relationship which belongs to one of the above four categories. We propose to utilize the concept of social relationships into the routing in CRAHNs.

\subsubsection{Application of social relationships in prediction of link duration}

Due to the fragility of the communication link, a link reliability prediction scheme is essential. However, most of the current studies ignored this aspect or just predict the link duration without considering the social relationship. Obviously, different relationships will result in different link durations, for example, the link between two friends should have a longer duration than that of two strangers. Therefore, we propose to combine the concept of social relationship with link duration prediction.

\subsubsection{Application of social relationships for relay selection}

Based on a large number of experiments and data statistics, we find out that the social relationship has a good stability. People used to meet with each other frequently have a larger probability to encounter with each other in the near future. In this paper, we intend to use the social relationship as a metric to make a routing decision and select the relay so as to make the transmission more effective.

\section{Analysis of link reliability}

In this section, we propose a prediction scheme which can judge the reliability of a link in a period of time. First, we give the following definitions.

Definition 2. (Reliable link) If there exists at least one common available channel between node $i$ and node $j$, and the available time of link $(i, j)$ on any common channel is longer than the actual time required for transmitting a message, then link $(i, j)$ is defined as a reliable one.

Definition 3. (Neighbors) The neighbors of node $i$ is a set of nodes which are within the transmission range $R_{0}$ of node $i$, denoted by $N_{i}$.

Assume at time $t_{0}$, node $i$ wants to transmit messages with size $Q$ to its destination $d_{i}$. Due to the limitation of power, a direct transmission is infeasible if node $i$ is far away from node $d_{i}$. Therefore, node $i$ has to select relays for help. Assume that node $i$ meets node $j$ at time $t$, the available common channel that node $i$ and node $j$ detected through spectrum sensing is $\Gamma_{i j}^{t}=$ $\{\operatorname{ch} 1, \ldots, \operatorname{ch} k, \ldots, c h \pi\}$, as illustrated in Figure 3 .

The effective bit rate of link $(i, j)$ on channel $k$ can be calculated by [4]:

$$
R_{i j}^{k}=B_{k} \log _{2}\left(1+\frac{S}{N}\right)=B_{k} \log _{2}\left(1+\frac{P_{i} h_{i j}}{N_{0} B_{k}}\right),
$$

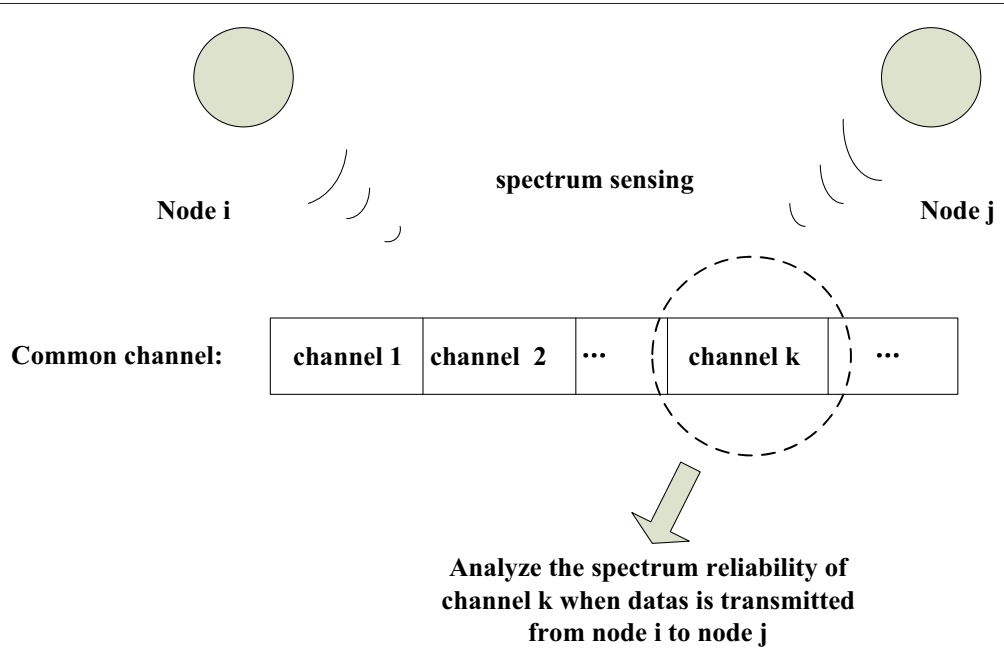

Figure 3 The common channel between node $i$ and node $j$. 
where $B_{k}$ is the bandwidth of channel $k, P_{i}$ is the transmission power of node $i, N_{0}$ is the noise spectral density, and $h_{i j}=\frac{\kappa}{d_{i j}^{\mu}}$ represents the path loss between $i$ and $j$, where $\kappa$ is the path loss constant, $\mu$ is the pass loss exponent, and $d_{i j}^{\mu}$ is the distance between $i$ and $j$.

Having the bit rate $R_{i j}^{k}$, we can get the time for transmitting data $Q$ from $i$ to $j$ :

$$
t_{i j}^{k}=\frac{Q}{R_{i j}^{k}}
$$

It is obvious that $t_{i j}^{k}$ is the actual transmission time needed for link $(i, j)$ transmitting data $Q$ on channel $k$. To transmit the data completely, the time link $(i, j)$ which remains available needs to be longer than $t_{i j}^{k}$. Next, we will predict the probability of link $(i, j)$ on channel $k$ remaining continuously available for time $t_{i j}^{k}$, denoted by $L_{i j}^{k}\left(t, t+t_{i j}^{k}\right)$, short for $L_{i j}^{k}$. If $L_{i j}^{k}$ is larger than a predefined threshold, link $(i, j)$ on channel $k$ is considered to be reliable. Based on the specific characteristics of CRAHNs, the interruption of a link can be classified into the following two categories according to its contributing factors.

Physical interruption: A link will be interrupted if the distance between two nodes becomes larger than the transmission range $R_{0}$. This kind of interruption is called physical interruption.

CR interruption: CR interruption is determined by spectrum availability. According to Definition 1, if the common channel $k$ between node $i$ and node $j$ becomes unavailable, then link $(i, j)$ on channel $k$ will be CR interrupted.

By the definition above, the prediction of $L_{i j}^{k}$ is determined by two aspects, the first one is $I_{i j}^{k}$ which is the probability that incurs no CR interruptions. The other aspect is $P_{i j}^{k}$, which is the probability without causing physical interruptions. The two concepts can be abstracted into a mathematical problem:

$$
\begin{aligned}
& I_{i j}^{k}\left(t, t+t_{i j}^{k}\right) \\
= & P\left\{\{ d _ { i , P U _ { k } } ( t ^ { \prime } ) > \rho _ { P k } \} \cap \{ d _ { j , P U _ { k } } ( t ^ { \prime } ) > \rho _ { P k } \} \cap \left\{d_{i, P U_{k}}\left(t^{\prime}\right)\right.\right. \\
& \left.>\rho_{S i}\right\} \cap\left\{d_{j, P U_{k}}\left(t^{\prime}\right)>\rho_{S j}\right\} \cap\left\{d_{i, P U_{B S}}\left(t^{\prime}\right)>\rho_{P_{B S}}\right\} \cap \\
& \left\{d_{j, P U_{B S}}\left(t^{\prime}\right)>\rho_{P_{B S}}\right\} \cap\left\{d_{i, P U_{B S}}\left(t^{\prime}\right)>\rho_{S i}\right\} \cap\left\{d_{j, P U_{B S}}\left(t^{\prime}\right)\right. \\
& \left.\left.>\rho_{S j}\right\}, t \leq t^{\prime} \leq t+t_{i j}^{k}\right\} \\
= & P\left\{\{ d _ { i , P U _ { k } } ( t ^ { \prime } ) > \operatorname { m a x } \{ \rho _ { P k } , \rho _ { S i } \} \} \cap \left\{d_{j, P U_{k}}\left(t^{\prime}\right)>\right.\right. \\
& \left.\max \left\{\rho_{P k}, \rho_{S j}\right\}\right\} \cap\left\{d_{i, P U_{B S}}\left(t^{\prime}\right)>\max \left\{\rho_{P_{B S}}, \rho_{S i}\right\}\right\} \\
& \left.\cap\left\{d_{j, P U_{B S}}\left(t^{\prime}\right)>\max \left\{\rho_{P_{B S}}, \rho_{S j}\right\}\right\}, t \leq t^{\prime} \leq t+t_{i j}^{k}\right\}
\end{aligned}
$$

$$
P_{i j}^{k}\left(t, t+t_{i j}^{k}\right)=P\left\{d_{i, j}\left(t^{\prime}\right)<R_{0}, t \leq t^{\prime} \leq t+t_{i j}^{k}\right\}
$$

It is well acknowledged that the social relationship between the two nodes has some effect on the prediction of $L_{i j}^{k}$. As a result, different prediction mechanisms are employed according to the friendship between the two nodes.

I. Close friends, Ordinary friends: Once the two nodes in this relationship encounter with each other, there is no doubt for them to have a pause for a chat. Consequently, we assume that the available time of link $(i, j)$ is long enough for data transmission. We set $L_{i j}^{k}=1$ directly in this situation.

II. Familiar strangers, Strangers: Nodes who have these relationships will not have any communication with each other when they encounter. The behaviors of each node are independent. As a result of this, prediction mechanisms are imperative to estimate $I_{i j}^{k}$ and $P_{i j}^{k}$.

Next we will explain the evaluation of $I_{i j}^{k}$ and $P_{i j}^{k}$ in the stranger cases.

According to our proposed mobility model, once two nodes encounter with each other, the motion states of each node can be classified into two phases according to the mobility model: (1) Keep the current velocity until the current epoch ends; (2) Change its velocity at the start of its next epoch, then keep the new velocity. Assume time for the first phase is $t_{1}$, and time for the second phase is $t_{2}$. Obviously, different prediction schemes should be employed in these two phases.

\subsection{Prediction of $I_{i j}^{k}\left(t_{1}\right)$ and $P_{i j}^{k}\left(t_{1}\right)$}

As mentioned above, $I_{i j}^{k}\left(t_{1}\right)$ is the probability for link $(i, j)$ on channel $k$ bringing no CR interruption within time $t_{1}$. If nodes $i$ and $j$ are not friends, the mobility patterns of the two nodes are deemed to be independent with each other. Therefore, $I_{i j}^{k}\left(t_{1}\right)$ can be expressed as:

$$
\begin{aligned}
I_{i j}^{k}\left(t_{1}\right)= & I_{i}^{k}\left(d_{i, P U_{k}}^{t}, t_{1}\right) \cdot I_{j}^{k}\left(d_{j, P U_{k}}^{t}, t_{1}\right) \cdot I_{i}^{B S}\left(d_{i, P U_{B S}}^{t}, t_{1}\right) \\
& \cdot I_{j}^{B S}\left(d_{j, P U_{B S}}^{t}, t_{1}\right)
\end{aligned}
$$

where $I_{i}^{k}\left(d_{i, P U_{k}}^{t}, t_{1}\right)$ and $I_{j}^{k}\left(d_{j, P U_{k}}^{t}, t_{1}\right)$ denote the probabilities for node $i$ and node $j$ bringing no interference to the $\mathrm{PU}_{k}$ within time $t_{1}$ given the initial distance $d_{i, P U_{k}}^{t}$ and $d_{j, P U_{k}}^{t}$, and $I_{i}^{B S}\left(d_{i, P U_{B S}}^{t}, t_{1}\right)$ and $I_{j}^{B S}\left(d_{j, P U_{B S}}^{t}, t_{1}\right)$ denote the probabilities for node $i$ and node $j$ bringing no interference to PU base station. The derivation of $I_{i}^{k}, I_{j}^{k}, I_{i}^{B S}$, and $I_{j}^{B S}$ is similar, and we take $I_{i}^{k}$ as an example to illustrate the derivation process. 
The nodes will keep their current velocity during time $t$ to $t+t_{1}$. Assume the current velocity is known to each node, taking node $i$ for example, the current speed is $v_{i}$ and the current direction is heading towards $C_{i}$. The position of $\mathrm{PU}_{k}$ is fixed at $P$. Assume the position of node $i$ is $A$ at time $t$. After time $t_{1}$, node $i$ arrives at $B$, and the current epoch ends. Let $A P=d_{0}, B P=d, \varphi_{i}$ denote the angle between $\overrightarrow{A B}$ and $\overrightarrow{A P}$, and $\varphi_{i} \in[0, \pi]$. Then $I_{i}^{k}\left(d_{0}, t_{1}\right)$ can be expressed as follows.

$$
I_{i}^{k}\left(d_{0}, t_{1}\right)= \begin{cases}1 & \text { satisfying (1) or (2) or (3) } \\ 0 & \text { otherwise }\end{cases}
$$

$$
\begin{aligned}
& \text { (1) } \varphi_{i} \in\left[\frac{\pi}{2}, \pi\right] \\
& \text { (2) } \varphi_{i} \in\left[0, \frac{\pi}{2}\right), d_{0} \cdot \sin \varphi_{i}>\max \left\{\rho_{P k}, \rho_{S i}\right\} \\
& \text { (3) } \varphi_{i} \in\left[0, \frac{\pi}{2}\right), d_{0} \cdot \sin \varphi_{i} \leq \max \left\{\rho_{P k}, \rho_{S i}\right\}, v_{i} \cdot t_{1}<d_{1}
\end{aligned}
$$

where $d_{1}=d_{0} \cdot \cos \varphi_{i}-\sqrt{\max \left\{\rho_{P k}, \rho_{S i}\right\}^{2}-\left(d_{0} \cdot \sin \varphi_{i}\right)^{2}}$. $P_{i j}^{k}\left(t_{1}\right)$ denotes the probability for nodes $i$ and $j$ remaining within the transmission range $R_{0}$ for time $t_{1}$. The mobility of the two nodes brings a huge computation complexity. Therefore, an approximate method is necessary. As the current velocity and location are known to each node. We can get the location of each node after $t_{1}$. Let the distance between $i$ and $j$ after $t_{1}$ is denoted as $d_{i j}^{t+t_{1}}$. We define that link $(i, j)$ is considered to be interrupted if $d_{i j}^{t+t_{1}}>R_{0}$, and vice versa.

$$
P_{i j}^{k}\left(t_{1}\right)= \begin{cases}1 & \text { if } d_{i j}^{t+t_{1}}<R_{0} \\ 0 & \text { otherwise }\end{cases}
$$

Therefore, we can obtain the probability for the link $(i, j)$ on channel $k$ remaining available in time $t_{1}$ for the stranger cases.

$$
L_{i j}^{k}\left(t_{1}\right)=I_{i j}^{k}\left(t_{1}\right) \cdot P_{i j}^{k}\left(t_{1}\right)
$$

\subsection{Prediction of $l_{i j}^{k}\left(t_{2}\right)$ and $P_{i j}^{k}\left(t_{2}\right)$}

At the start of $t_{2}$, each node makes a choice of a new velocity. Different from that in the $t_{1}$ period, the newly chosen velocity is unknown to each node. Thus it is necessary to consider all the eventualities. Similarly with $I_{i j}^{k}\left(t_{1}\right)$ in the former subsection, $I_{i j}^{k}\left(t_{2}\right)$ can be expressed as:

$$
\begin{aligned}
I_{i j}^{k}\left(t_{2}\right)= & I_{i}^{k}\left(d_{i, P U_{k}}^{t+t_{1}}, t_{2}\right) \cdot I_{j}^{k}\left(d_{j, P U_{k}}^{t+t_{1}}, t_{2}\right) \cdot I_{i}^{B S}\left(d_{i, P U_{B S}}^{t+t_{1}}, t_{2}\right) \\
& \cdot I_{j}^{B S}\left(d_{j, P U_{B S}}^{t+t_{1}}, t_{2}\right),
\end{aligned}
$$

Taking $I_{i}^{k}\left(d_{i, P U_{k}}^{t+t_{1}}, t_{2}\right)$ as an example, it can be expressed as follows.

$$
I_{i}^{k}\left(d_{i, P U_{k}}^{t+t_{1}}, t_{2}\right)=\sum_{m=1}^{\omega} I_{i, m}^{k}\left(d_{i, P U_{k}}^{t+t_{1}}, t_{2}\right) \cdot \chi_{i}^{m},
$$

where $\chi_{i}^{m}$ denotes the probability for node $i$ to move towards zone $m$. $I_{i, m}^{k}$ denotes the probability of node $i$ moving towards zone $m$ for time $t_{2}$ without generating any interference to $\mathrm{PU}_{k}$. As shown in Figure 4, the position of $\mathrm{PU}_{k}$ is denoted by $P$. The initial location of node $i$ at time $t+t_{1}$ is $A$. After a period of time $t_{2}$, node $i$ arrives at $B$, where $A P=d_{0}, B P=d . \varphi_{m}^{k}$ denotes the angle between $\overrightarrow{A B}$ and $\overrightarrow{A P}$, and $\varphi_{m}^{k} \in[0, \pi]$. The subscript $m$ of

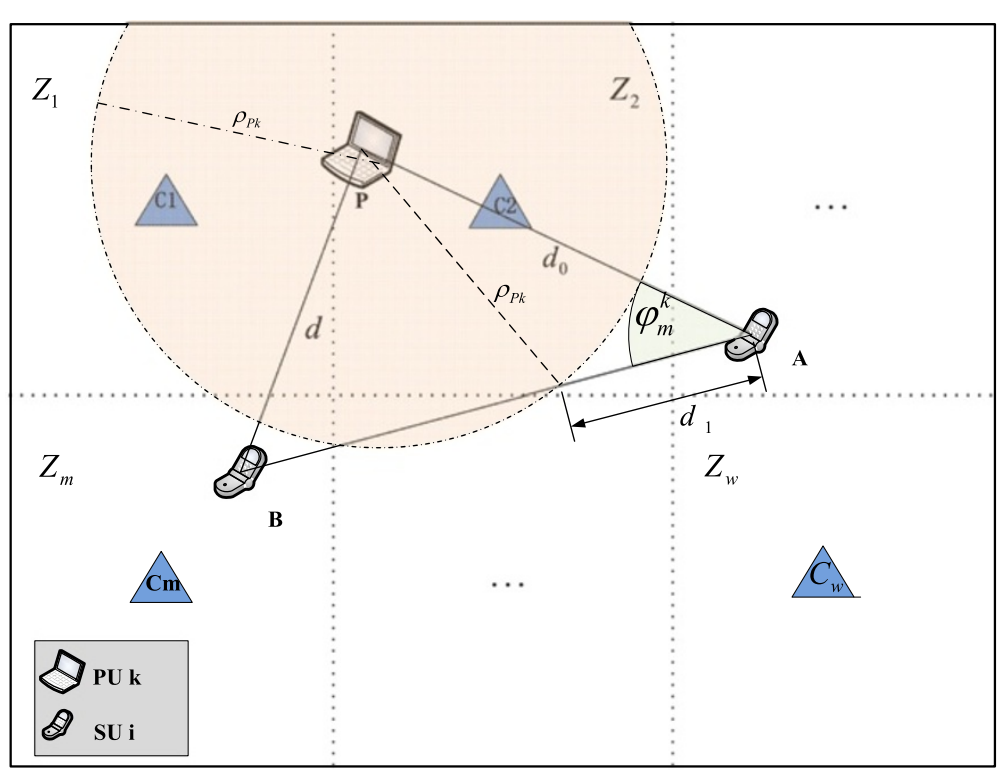

Figure 4 Prediction of $l_{i}^{k}$. 
$\varphi_{m}^{k}$ means node $i$ moves towards zone $m$, and the superscript $k$ denotes the distance is measured between node $i$ and $\mathrm{PU}_{k}$. The value of $I_{i m}^{k}\left(d_{0}, t_{2}\right)$ is similar to $I_{i}^{k}\left(d_{0}, t_{1}\right)$.

$$
I_{i, m}^{k}\left(d_{0}, t_{2}\right)= \begin{cases}1 & \text { satisfying (1) or (2) or (3) } \\ \frac{\frac{d_{1}}{t_{2}}-v_{\min }}{v_{\max }-v_{\min }} & \text { otherwise }\end{cases}
$$

(1) $\varphi_{m}^{k} \in\left[\frac{\pi}{2}, \pi\right]$

(2) $\varphi_{m}^{k} \in\left[0, \frac{\pi}{2}\right), d_{0} \cdot \sin \varphi_{m}^{k}>\max \left\{\rho_{P k}, \rho_{S i}\right\}$

(3) $\varphi_{m}^{k} \in\left[0, \frac{\pi}{2}\right), d_{0} \cdot \sin \varphi_{m}^{k} \leq \max \left\{\rho_{P k}, \rho_{S i}\right\}, v_{\max } \cdot t_{2}<d_{1}$

where $d_{1}=d_{0} \cdot \cos \varphi_{m}^{k}-\sqrt{\max \left\{\rho_{P k}, \rho_{S i}\right\}^{2}-\left(d_{0} \cdot \sin \varphi_{m}^{k}\right)^{2}}$, as shown in Figure 4.

$P_{i j}^{k}\left(t_{2}\right)$ denotes the probability for nodes $i$ and $j$ remaining within the transmission range $R_{0}$ for time $t_{2}$. As illustrated in Figure 5, the initial positions of $i$ and $j$ are denoted as $A$ and $B$, respectively, at $t+t_{1}$, and the distance between $i$ and $j$ is $d_{i j}^{t+t_{1}}$, which can be obtained in the last subsection. After time $t_{2}, i$ and $j$ arrived at positions $A^{\prime}$ and $B^{\prime}$, respectively, and the distance between them turns to be $d_{i j}^{t+t_{1}+t_{2}}$. As both nodes choose a new direction heading towards one of the zones. Assume node $i$ is walking towards zone $m$, and $\phi_{m}^{i}$ is the angle between vectors $\overrightarrow{A A^{\prime}}$ and $\overrightarrow{A B}$. Similarly, $\phi_{n}^{j}$ denotes the angle between vectors $\overrightarrow{B B^{\prime}}$ and $\overrightarrow{B A}$ when node $j$ is walking towards zone $n$.
The probability $P_{i j}^{k}\left(t_{2}\right)$ can be expressed as follows.

$$
P_{i j}^{k}\left(t_{2}\right)=\sum_{m=1}^{\omega} \sum_{n=1}^{\omega} P_{i, m}^{j, n}\left(d_{i j}^{t+t_{1}}, t_{2}\right) \cdot \chi_{i}^{m} \cdot \chi_{j}^{n},
$$

where $P_{i, m}^{j, n}\left(d_{i j}^{t+t_{1}}, t_{2}\right)$ denotes the approximate probability for nodes $i$ and $j$ to keep in the transmission range $R_{0}$ continuously within time $t_{2}$ when $i$ is walking towards zone $m$ and $j$ towards zone $n$, given the initial distance $d_{i j}^{t+t_{1}}$. Similarly, an approximate method is adopted in evaluating the value of $P_{i, m}^{j, n}\left(d_{i j}^{t+t_{1}}, t_{2}\right)$. We set the velocity of nodes $i$ and $j$ to be the mean value. In this situation, we can estimate the distance between $i$ and $j$ after $t_{2}$, denoted as $\widetilde{d}_{i j}^{t+t_{1}+t_{2}}$. If $\tilde{d}_{i j}^{t+t_{1}+t_{2}}>R_{0}$, link $(i, j)$ is considered to be interrupted, and vice versa.

From Figure 5, we can obtain

$$
\begin{aligned}
& A^{\prime} E=d_{i j}^{t+t_{1}}-E\left[v_{i}\right] \cdot t_{2} \cdot \cos \phi_{m}^{i}-E\left[v_{j}\right] \cdot t_{2} \cdot \cos \phi_{n}^{j} \\
& B^{\prime} E= \begin{cases}E\left[v_{i}\right] \cdot t_{2} \cdot \sin \phi_{m}^{i}-E\left[v_{j}\right] \cdot t_{2} \cdot \sin \phi_{n}^{j} & \text { case } 1 \\
\left|E\left[v_{i}\right] \cdot t_{2} \cdot \sin \phi_{m}^{i}+E\left[v_{j}\right] \cdot t_{2} \cdot \sin \phi_{n}^{j}\right| & \text { case } 2\end{cases}
\end{aligned}
$$

case $1: i, j$ are walking towards the same side of line $A B$. case $2: i, j$ are walking towards different sides of line $A B$.

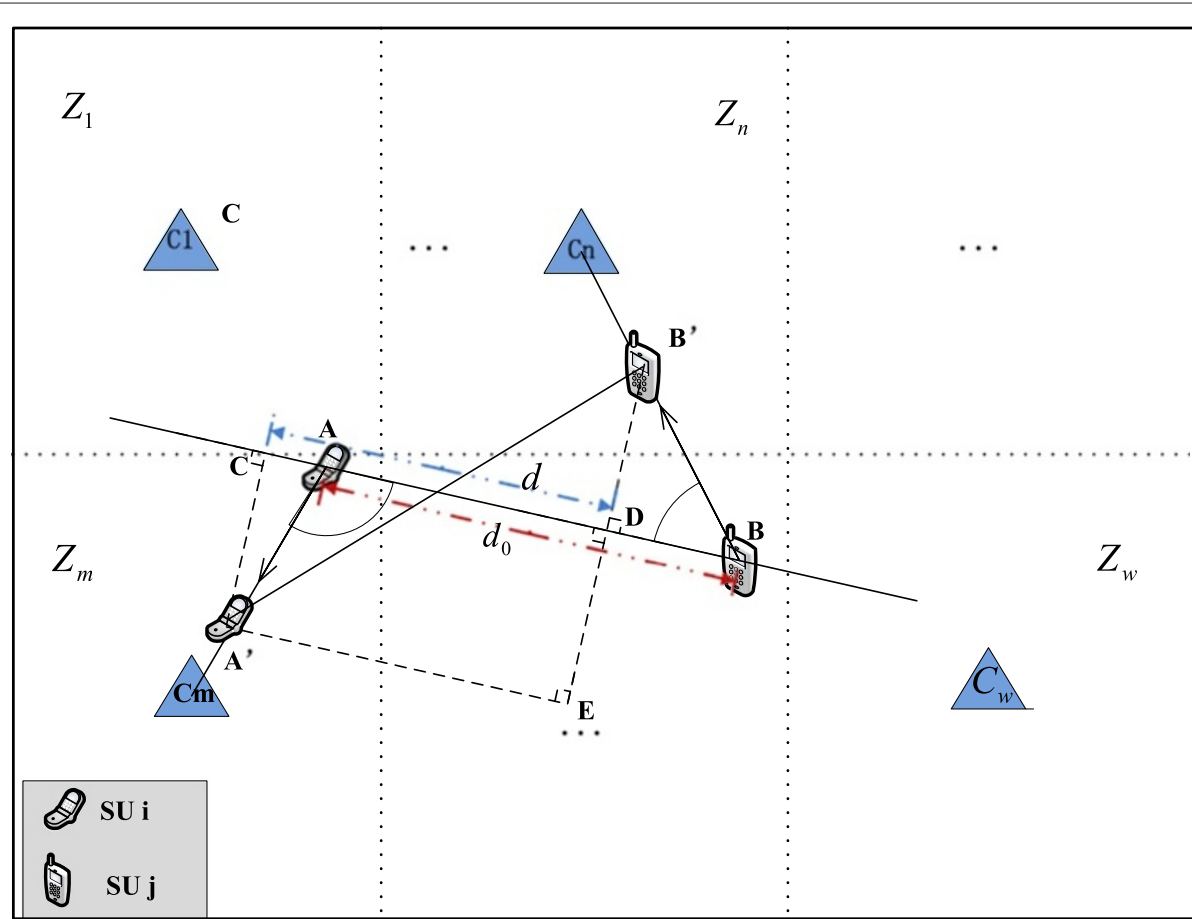

Figure 5 Prediction of $P_{i j}^{k}$. 
In right-angled triangle $\triangle A^{\prime} B^{\prime} E$, we can get

$$
\tilde{d}_{i j}^{t+t_{1}+t_{2}}=d=A^{\prime} B^{\prime}=\sqrt{A^{\prime} E^{2}+B^{\prime} E^{2}}
$$

Then we can obtain $P_{i m}^{j n}\left(d_{i j}^{t+t_{1}}\right)$ according to the comparison with $R_{0}$.

$$
P_{i, m}^{j, n}\left(d_{i j}^{t+t_{1}}, t_{2}\right)= \begin{cases}1 & \text { if } \tilde{d}_{i j}^{t+t_{1}+t_{2}}<R_{0} \\ 0 & \text { otherwise }\end{cases}
$$

Therefore, we can obtain the probability that link $(i, j)$ on channel $k$ remains available in time $t_{2}$ in the stranger cases.

$$
L_{i j}^{k}\left(t_{2}\right)=I_{i j}^{k}\left(t_{2}\right) \cdot P_{i j}^{k}\left(t_{2}\right)
$$

\subsection{Analysis of link reliability}

From the analysis above, we can obtain the probability for link $(i, j)$ on channel $k$ remaining available from time $t_{0}$ to $t_{0}+t_{i j}^{k}$.

$$
L_{i j}^{k}\left(t_{i j}^{k}\right)= \begin{cases}1 & \text { if } i, j \text { are friends } \\ L_{i j}^{k}\left(t_{1}\right) \cdot L_{i j}^{k}\left(t_{2}\right) & \text { if } i, j \text { are not friends }\end{cases}
$$

If $L_{i j}^{k}$ is larger than a threshold $L^{*}$, link $(i, j)$ on channel $k$ is said to be reliable, and vice versa. Link $(i, j)$ is considered to be reliable if there exists at least one reliable channel between them, and it is necessary to choose the most reliable channel from all the available ones. The reliable link prediction algorithm is detailed in Algorithm 1.

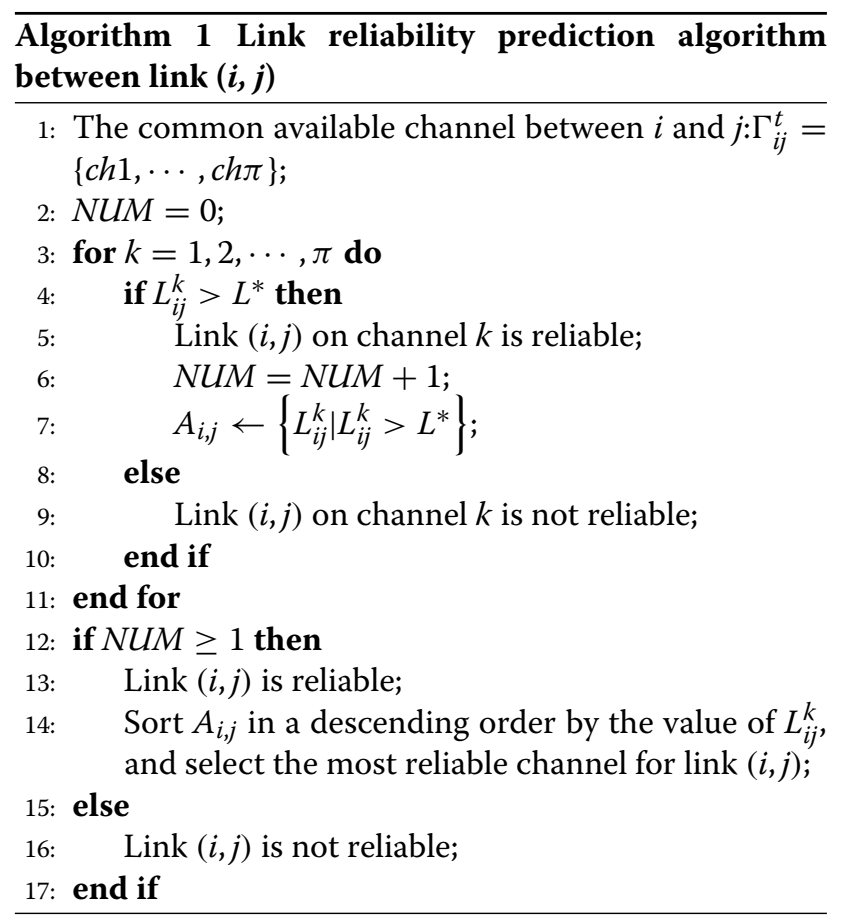

\section{Routing scheme}

In the previous section, a link reliability prediction algorithm has been proposed. Based on this algorithm, we present a new social-based opportunistic routing scheme, named SoRoute, which selects relays in a reliable and efficient way.

Based on a larger number of observations, we can conclude that people who used to meet frequently have the potential to meet each other with a larger probability [33]. Therefore, node $i$ will select the node that had more frequent encounters with the destination as a relay to forward the message. This will result in a larger probability to send the message to the destination. The complete process of the social-based opportunistic forwarding algorithm is described in Algorithm 2.

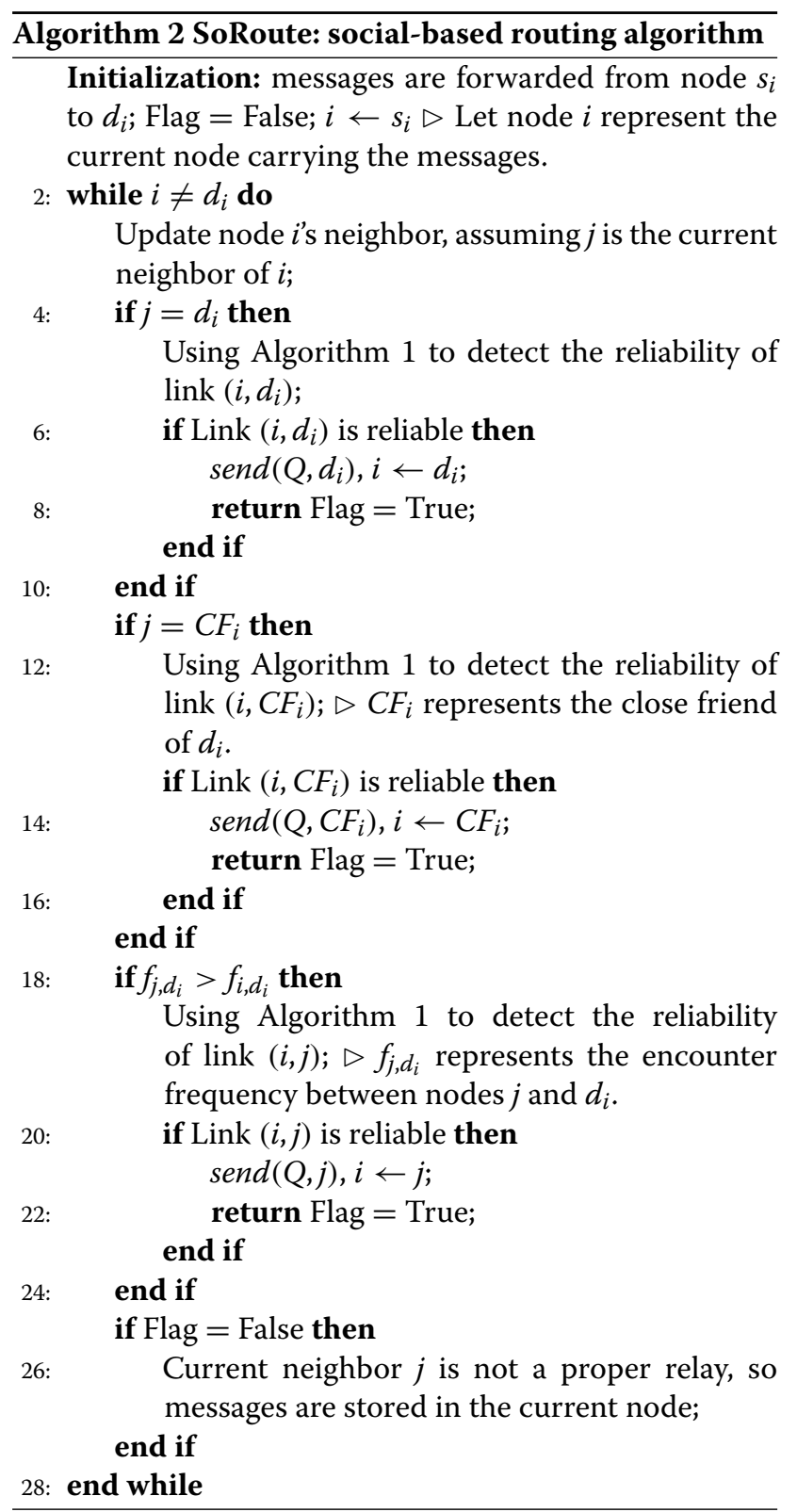


Assume that node $i$ carries the data destined to node $d_{i}$. The opportunistic forwarding strategy of node $i$ can be divided into the following stages:

(1) Spectrum sensing: Once node $i$ meets one of its neighbors $j, j$ sends its location, current velocity, and social relationship information through a common control channel (CCC) to $i$. We also assume that the SUs are able to access a geo-database to obtain the PUs' location. Then node $i$ can detect the common available channels with node $j$.

(2) Social-based relay selection: Node $i$ checks the relationship between node $j$ and the destination. If node $j$ is the destination or one of the destination's close friends, or has a destination encounter frequency greater than its own encounter frequency with the destination, node $i$ will further check whether the link to node $j$ is reliable (see step (3)). Otherwise, it will stop the process.

(3) Reliable path selection: Node $i$ computes link reliability $L_{i j}^{k}$ of every common available channel with $j$ according to the link reliability detection algorithm. Then node $i$ can judge whether node $j$ is a reliable relay. If node $j$ is a reliable relay, then node $i$ relays the message to node $j$.

Assume that node $j$ is selected as the most suitable relay for node $i$ by applying the above routing algorithm, then $j$ conducts the same operation accordingly until the message is sent to the destination.

\section{Simulation}

In this section, we evaluate the performance of the proposed social-based routing scheme in CRAHNs. First, we will verify the correctness of the proposed link reliability prediction algorithm. Then the proposed routing scheme is compared with the state-of-the-art DTN routing schemes: First Contact, Direct Delivery, MaxProp, and Prophet.

\subsection{Validation of the proposed link reliability prediction algorithm}

In order to verify the correctness of our link reliability prediction algorithm, we compare the results of our prediction algorithm with that of the actual simulation, in which each node moves according to the mobility model we proposed in section 3 . The simulation environment is a two-dimensional space in an area of 4,500 × 3,400 m. Then we divided the whole area into six parts evenly (two rows $\times$ three columns) representing six different zones. For simplicity, only one PU and two SUs are placed in the simulation. The probability of the two SUs to move towards each zone is set to be $[0.30,0.16,0.14,0.15,0.10,0.15]$ and

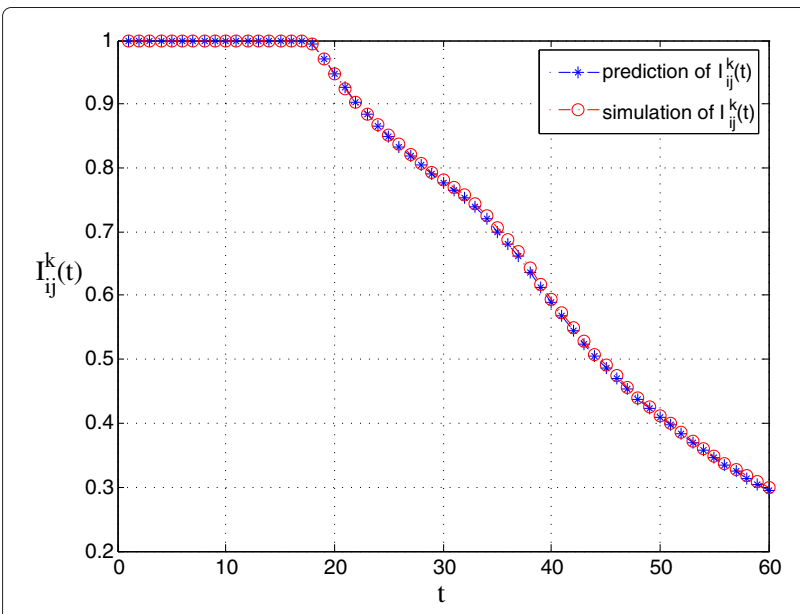

Figure $6 l_{i j}^{k}(t)$ comparison of prediction and simulation.

$[0.10,0.05,0.40,0.05,0.10,0.30]$, respectively. The radio transmission range is set to be $150 \mathrm{~m}$, and the interference range of PUs and SUs are set to be 300 and $200 \mathrm{~m}$, respectively. The speed of SUs is uniformly distributed between 0 and $8 \mathrm{~m} / \mathrm{s}$.

Figures 6, 7, 8 compare the results of the prediction algorithm and the simulation. Red lines represent the simulation results, and blue lines denote the prediction algorithm results.

Figure 6 shows the results of $I_{i j}^{k}(t)$. It is clear from the figure that the red lines are fairly close with the blue ones, which means our prediction of the probability with no CR interruption is quite accurate.

Figure 7 gives the results of $P_{i j}^{k}(t)$, which is the probability of nodes $i$ and $j$ remaining in the transmission range of each other within time $t$. From the figure, we can see that there is about $30 \%$ deviation.

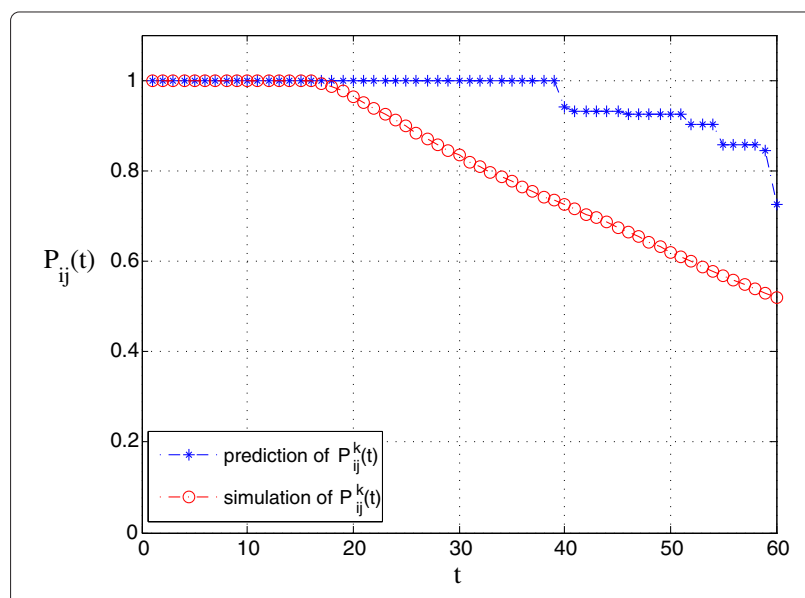

Figure $7 P_{i j}^{k}(t)$ comparison of prediction and simulation. 


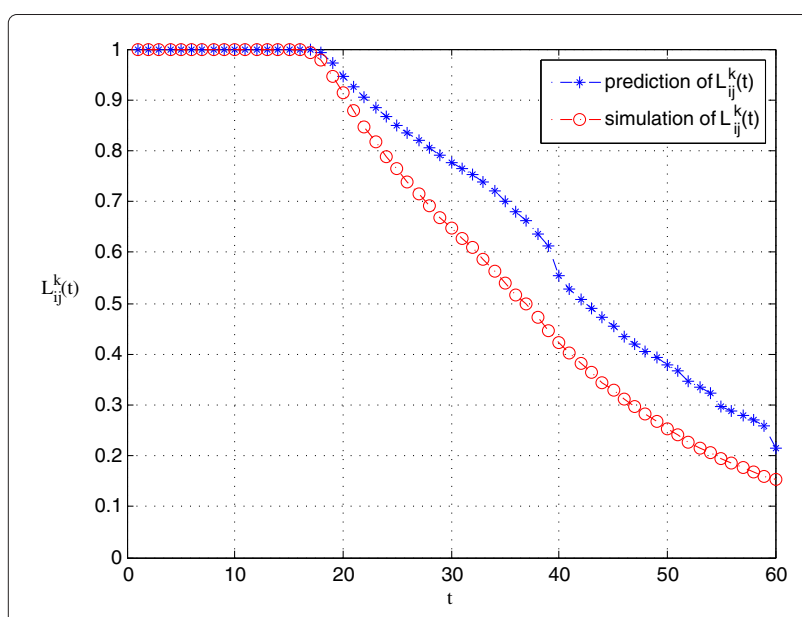

Figure $8 L_{i j}^{k}(t)$ comparison of prediction and simulation.
However, the final results of link reliability $L_{i j}^{k}$ is the product of the former two parameters, and the results are shown in Figure 8. We can observe that the two lines are close enough with a maximum deviation of $13 \%$. Therefore, we can draw the conclusion that our proposed scheme is precise enough to predict the link reliability in actual mobile environments.

\subsection{Statistics of the social relationship between nodes}

To evaluate the performance of our proposed SoRoute, we implemented the proposed algorithms in the simulator [34]. Figure 9 is a screen shot of the ONE simulator. Thirty nodes are involved in our experiments based on the mobility model proposed in section 3. Detailed parameters of network simulation environment are summarized in Table 1.

As discussed before, the social relationship between two nodes can be classified into four categories according to their encounter durations and frequencies. Figure 10 and Figure 11 show the statistical encounter durations and

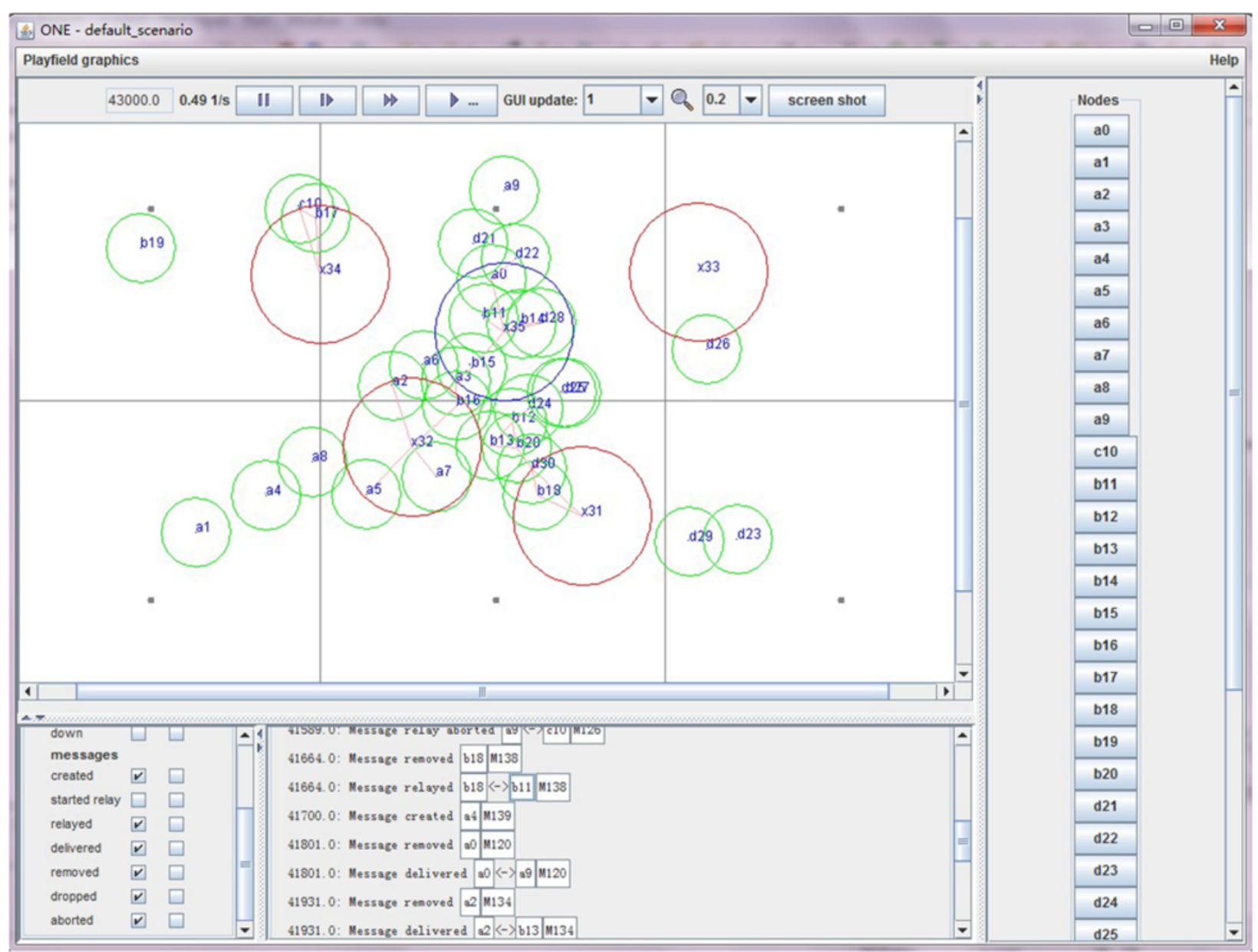

Figure 9 A screen shot of the ONEs. 
Table 1 Simulation parameters

\begin{tabular}{ll}
\hline Parameters & Value \\
\hline Simulator & The ONEs \\
Simulation environment & $(4,500 \mathrm{~m}, 3,400 \mathrm{~m})$ \\
$R_{0}:$ radio transmission range & $150 \mathrm{~m}$ \\
$\rho_{P}, \rho_{S}$ : interference range of PUs and SUs & $300 \mathrm{~m}$ \\
Speed distribution & Uniform $[0,8 \mathrm{~m} / \mathrm{s}]$ \\
Direction distribution & Towards the landmark of \\
& each zone \\
Epoch length & $60 \mathrm{~s}$ \\
Number of primary base station & 1 \\
Number of PU & 4 \\
Number of SU & 30 \\
Transmission speed & $384 \mathrm{kBps}$ \\
Buffer size & $80 \mathrm{M}$ \\
Scenario endtime & $12 \mathrm{~h}$ \\
\hline
\end{tabular}

frequencies of these nodes within $12 \mathrm{~h}$. Then Figure 12 shows the classification results of these nodes. The red triangles denote the node relationship of close friends, which are very sparse. On the contrary, the blue plus signs represent the strangers that are quite dense in the figure. The green asterisks and the pink circles denote the familiar strangers and the ordinary friends, respectively. The above statistical results are consistent with the reality.

\subsection{Performance of the proposed social-based routing scheme}

In this section, we compare the performance of our SoRoute with several alternative routing schemes based on the simulation.

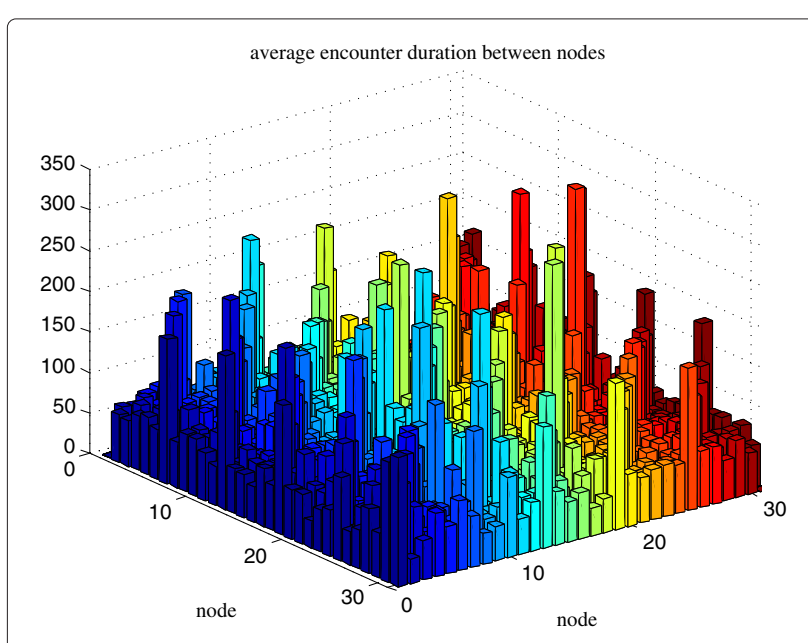

Figure 10 Statistics of encounter duration between nodes.

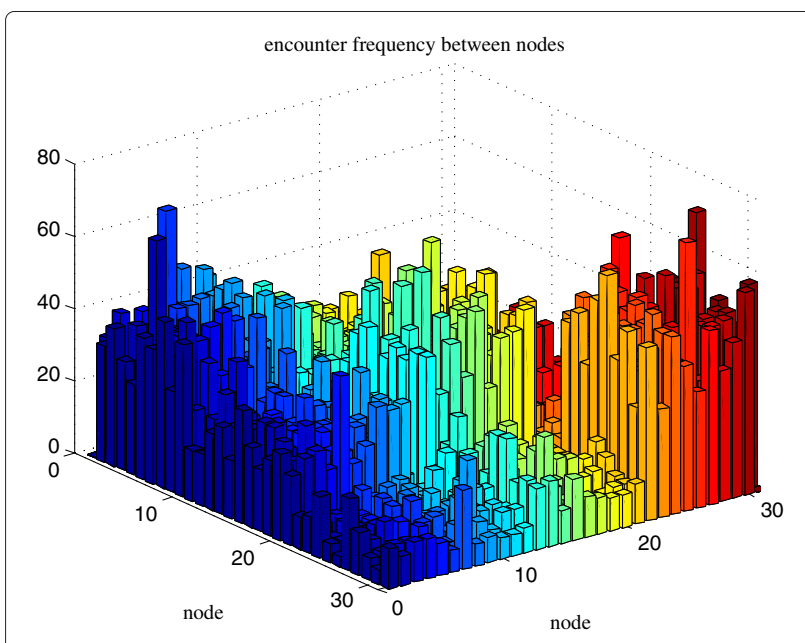

Figure 11 Statistics of encounter frequency between nodes.

- Direct delivery routing: This routing scheme is the simplest one. In this algorithm, messages are forwarded only when they encounter with the destination node.

- First contact routing: In this scheme [35], messages are forwarded to a randomly chosen contact or to the first available contact if none of them are connected at the time of message arrival.

- Maxprop routing: MaxProp [20] is based on prioritizing the schedule of packets transmitted to other peers and the schedule of packets to be dropped. These priorities are based on the path likelihoods to peers according to the historical data and also on several complementary mechanisms, including acknowledgments, a head-start for new packets, and lists of previous intermediaries.

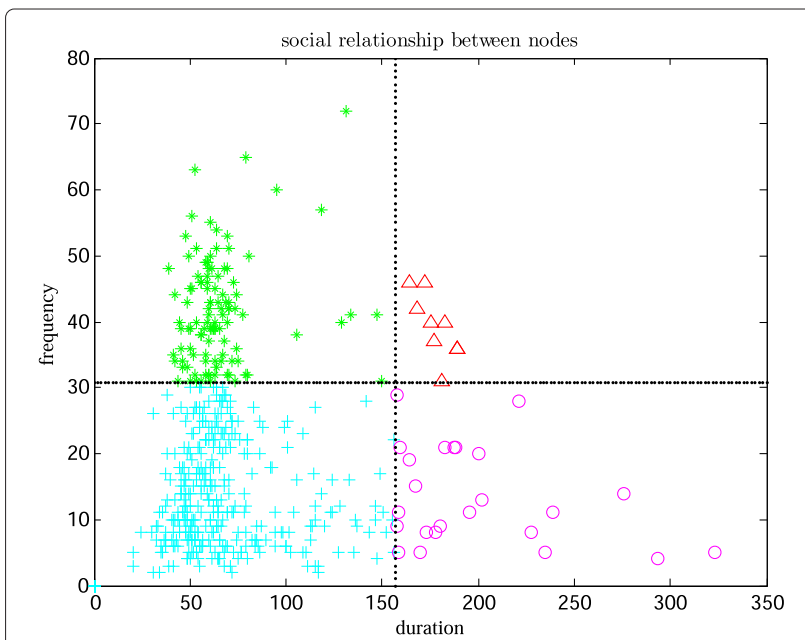

Figure 12 Statistics of the social relationship between nodes. 


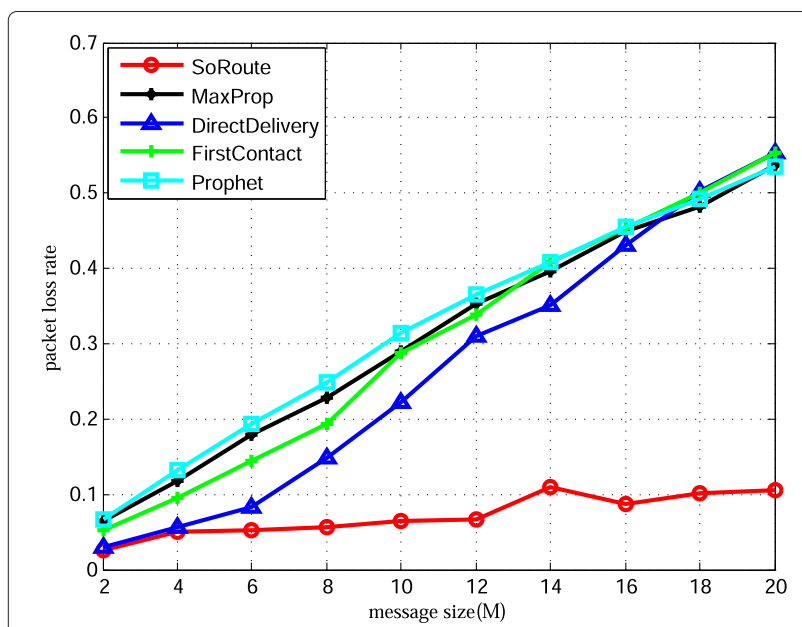

Figure 13 Packet loss ratio with increasing message size.

- Prophet routing: Prophet [36] is a DTN routing protocol aiming at using knowledge obtained from past encounters with other nodes to optimize packet delivery. Each node keeps a vector of delivery predictability estimates and uses it to decide whether an encountered node were a better carrier for a DTN packet.

We consider four important metrics to compare the performance of our social-based forwarding strategy with the above routing schemes:

- Packet loss rate: It refers to the ratio of the aborted messages to the total number of messages during the transmission of each hop .

- Packet delivery rate: It refers to the ratio of the successfully delivered messages to the total number of created messages at the end of an experiment.

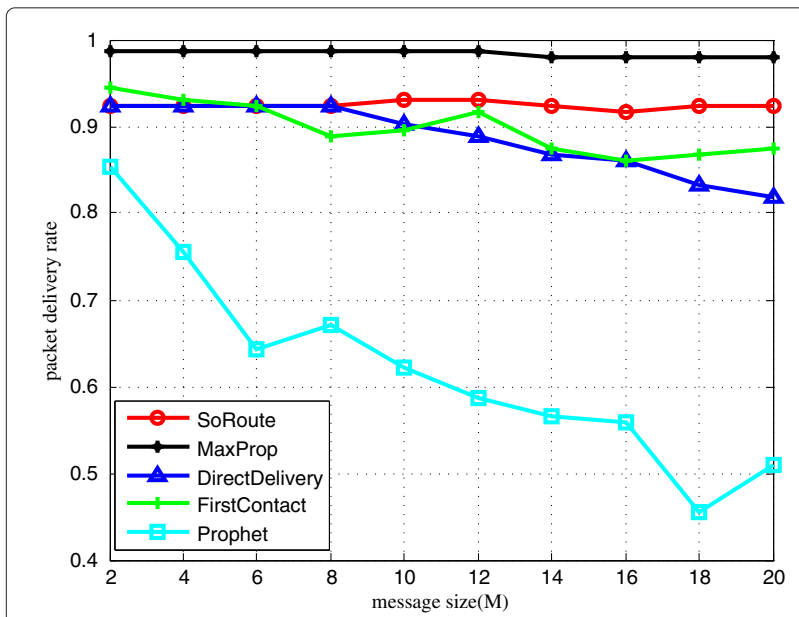

Figure 14 Packet delivery rate with increasing message size.

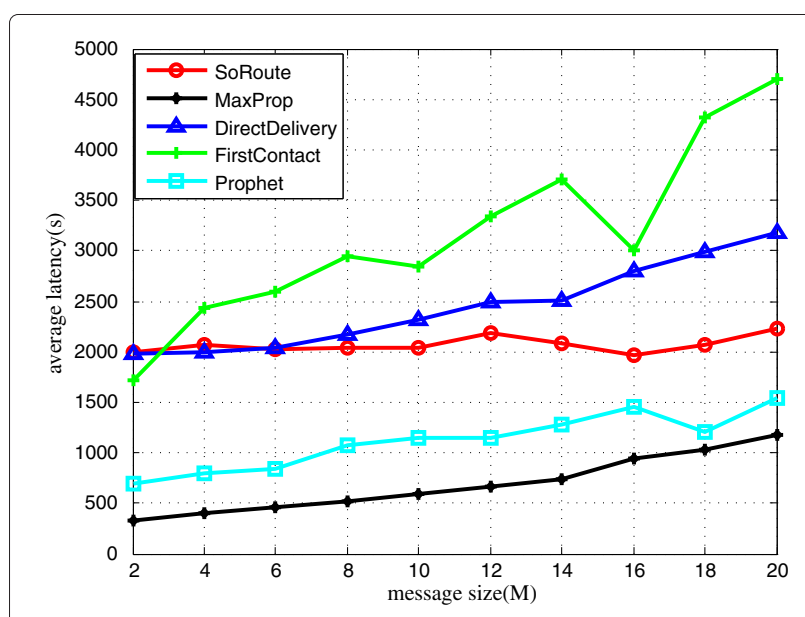

Figure 15 Average latency with increasing message size.

- Average latency: An end-to-end delay refers to the delay for a message to be received at its destination. Here we consider the average value of delays for all successfully delivered messages during the experiment time.

- Overhead ratio: It is a metric of measuring the system costs, and it's defined as follows: $\underline{\text { relayed messages - delivered messages }}$

Figures 13, 14, 15, 16 show the performances as a function of message size. From Figure 13, it can be seen that SoRoute has the lowest packet loss rate. That means the chosen link in our routing scheme is the most reliable one especially when the message size is large. This is due to the fact that our routing scheme has a process of link reliability prediction. Besides, we also observe that the packet loss rate increases with message size. This trend is well understood because the larger the message size is, the longer

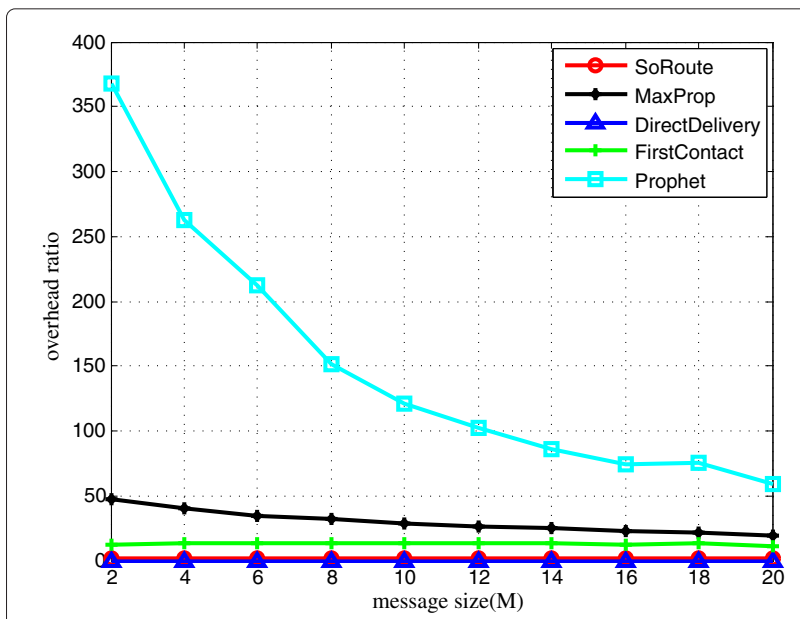

Figure 16 Overhead ratio with increasing message size. 
time it takes to be transmitted, then the link is easier to break.

Figure 14 illustrates that only the MaxProp scheme outperforms our SoRoute in packet delivery ratio. More precisely, our routing scheme has a stable packet delivery ratio of $92.3 \%$, with only $5.7 \%$ degradation compared to the MaxProp.

The results of the average end-to-end delay are shown in Figure 15. Maxprop and Prophet, in which multiple message copies coexisting in the networks help spread the messages, have the minimum delay. In contrast, there exists only a single copy of messages in the network for First Contact, Direct Delivery, and our SoRoute. It is obvious that SoRoute has the best performance among the three single-copy schemes.

Finally, the overhead ratio is illustrated in Figure 16; it can be seen from the figure that SoRoute has quite a low overhead. In contrast, the overheads of Maxprop and Prophet are much higher because of their multicopy characteristics. These results prove that SoRoute is a reliable, low-overhead, and low-latency routing algorithm.

\section{Conclusions}

In this paper, we propose a social-based routing scheme for CRAHNs. The proposed routing scheme utilizes the social relationships between people to select relays in a reliable and effective way. The simulation results show that our routing scheme achieves a much better performance in terms of packet loss rate and overhead ratio than the existing routing schemes such as Direct Delivery, First Contact, MaxProp, and Prophet. Our future work involves improving the mobility model and validate it using more realistic data.

\section{Competing interests}

The authors declare that they have no competing interests.

\section{Acknowledgements}

The authors would like to thank the support from the National Natural Science Foundation of China (Grant No. 61272505, 61172074, and 61371069) and the National Science Foundation of the US (CNS-1162057).

\section{Author details}

${ }^{1}$ School of Electronics and Information Engineering, Beijing Jiaotong University, 100044 Beijing, China. ${ }^{2}$ Department of Electrical Engineering and Computer Science, the Catholic University of America, 20064 Washington DC, USA.

Received: 27 July 2014 Accepted: 7 October 2014

Published: 25 November 2014

\section{References}

1. IF Akyildiz, W-Y Lee, MC Vuran, S Mohanty, Next generation/dynamic spectrum access/cognitive radio wireless networks: a survey. Comput. Netw. 50(13), 2127-2159 (2006)

2. W Li, X Cheng, T Jing, Y Cui, K Xing, W Wang, Spectrum assignment and sharing for delay minimization in multi-hop multi-flow crns. IEEE J. Selected Areas Commun. (JSAC) 31(11), 2483-2493 (2013)

3. $\mathrm{H} \mathrm{Li}, \mathrm{X}$ Cheng, $\mathrm{KLi}, \mathrm{XX}$ Xing, $\mathrm{T}$ Jing, in Proceedings. INFOCOM Mini-Conference, 2013. Utility-based cooperative spectrum sensing scheduling in cognitive radio networks (Italy, 2013), pp. 165-169
4. W Li, X Cheng, T Jing, X Xing, in Proceedings. INFOCOM, 2013. Cooperative multi-hop relaying via network formation games in cognitive radio networks (Italy, 2013), pp. 971-979

5. X Xing, T Jing, W Cheng, Y Huo, X Cheng, Spectrum prediction in cognitive radio networks. IEEE Wireless Commun. 20(2), 90-96 (2013)

6. X Xing, T Jing, Y Huo, H Li, X Cheng, in Proceedings. INFOCOM, 2013. Channel quality prediction based on bayesian inference in cognitive radio networks (Italy, 2013), pp. 1465-1473

7. T Jing, X Chen, Y Huo, X Cheng, in Proceedings. IEEE INFOCOM, 2012 Achievable transmission capacity of cognitive mesh networks with different media access control (USA, 2012), pp. 1764-1772

8. M Song, $C X$ in, $Y$ Zhao, $X$ Cheng, Dynamic spectrum access: from cognitive radio to network radio. IEEE Wireless Commun. 19(1), 23-29 (2012)

9. IF Akyildiz, W-Y Lee, KR Chowdhury, Crahns: Cognitive radio ad hoc networks. Ad Hoc Netw. 7(5), 810-836 (2009)

10. Z Zhang, Routing in intermittently connected mobile ad hoc networks and delay tolerant networks: overview and challenges. IEEE Commun. Surv. Tutor. 8(1), 24-37 (2006)

11. J Huang, S Wang, X Cheng, M Liu, Z Li, B Chen, in IEEE Transaction on Parallel Distributed Systems (TPDS). Mobility-assisted routing in intermittently connected mobile cognitive radio networks, (2014), pp. 2956-2968

12. S Chen, K Nahrstedt, Distributed quality-of-service routing in ad hoc networks. IEEE J on Selected Areas Communication 17(8), 1488-1505 (1999)

13. A Vahdat, D Becker, Epidemic routing for partially connected ad hoc networks. Technical report, Technical Report CS-200006, Duke University (2000)

14. P Hui, J Crowcroft, E Yoneki, Bubble rap: social-based forwarding in delay-tolerant networks. IEEE Transaction on Mobile Computing 10(11), 1576-1589 (2011)

15. EM Daly, M Haahr, in Proceedings. The 8th ACM International Symposium on Mobile Ad Hoc Networking and Computing. Social network analysis for routing in disconnected delay-tolerant manets (ACM, 2007), pp. 32-40

16. S Wang, M Liu, X Cheng, Z Li, J Huang, B Chen, Opportunistic routing in intermittently connected mobile p2p networks. IEEE J on Selected Areas Communication 31(9), 369-378 (2013)

17. S Wang, M Liu, X Cheng, M Song, Routing in pocket switched networks. IEEE Wireless Commun. 19(2), 67-73 (2012)

18. H Zhu, M Dong, S Chang, Y Zhu, M Li, X Shen, in Proceedings. INFOCOM 2013 Zoom: scaling the mobility for fast opportunistic forwarding in vehicular networks, (2013), pp. 2832-2840

19. T Spyropoulos, K Psounis, CS Raghavendra, in Proceedings. The Fifth Annual IEEE International Conference On Pervasive Computing and Communications Workshops, 2007. Spray and focus: efficient mobility-assisted routing for heterogeneous and correlated mobility, (2007), pp. 79-85

20. J Burgess, B Gallagher, D Jensen, BN Levine, in Proceedings. INFOCOM 2006. 25th IEEE International Conference on Computer Communications. Maxprop: Routing for vehicle-based disruption-tolerant networks (Spain, 2006), pp. 1-11

21. P-C Cheng, J-T Weng, L-C Tung, KC Lee, M Gerla, J Haerri, in Proceedings. The 1st International Symposium on Vehicular Computing Systems (ISVCS'08). Geodtn+ nav: a hybrid geographic and dtn routing with navigation assistance in urban vehicular networks (Dublin, Ireland, July 2008)

22. F Khadar, T Razafindralambo, in NETWORKING 2009. Performance evaluation of gradient routing strategies for wireless sensor networks (Springer, Berlin, 2009), pp. 535-547

23. S Srinivasa, S Krishnamurthy, in Proceedings. The 6th Annual IEEE Communications Society Conference On Sensor, Mesh and Ad Hoc Communications and Networks, 2009. Crest: An opportunistic forwarding protocol based on conditional residual time, (2009), pp. 1-9

24. E Bulut, BK Szymanski, in Proceedings. Global Telecommunications Conference (GLOBECOM 2010), 2010. Friendship based routing in delay tolerant mobile social networks (IEEE, USA, 2010), pp. 1-5

25. W Gao, Q Li, B Zhao, G Cao, in Proceedings. The Tenth ACM International Symposium on Mobile Ad Hoc Networking and Computing. Multicasting in delay tolerant networks: a social network perspective (ACM, 2009), pp. 299-308

26. Q Han, Y Bai, L Gong, W Wu, Link availability prediction-based reliable routing for mobile ad hoc networks. Commun. IET 5(16), 2291-2300 (2011). doi:10.1049/iet-com.2010.0946 
27. S Jiang, D He, J Rao, in Proceedings. IEEE INFOCOM 2001. Twentieth Annual Joint Conference of the IEEE Computer and Communications Societies, vol. 3. A prediction-based link availability estimation for mobile ad hoc networks, (2001), pp. 1745-17523. doi:10.1109/INFCOM.2001.916672

28. S Jiang, An enhanced prediction-based link availability estimation for manets. IEEE Trans Commun. 52(2), 183-186 (2004). doi:10.1109/TCOMM.2003.822739

29. Q Guan, FR Yu, S Jiang, G Wei, Prediction-based topology control and routing in cognitive radio mobile ad hoc networks. IEEE Trans on Vehic. Technol. 59(9), 4443-4452 (2010). doi:10.1109/TVT.2010.2069105

30. X Cheng, A Thaeler, G Xue, D Chen, in Proceedings. IEEE INFOCOM, 2004, vol. 4. Tps: a time-based positioning scheme for outdoor sensor networks (HK, 2004), pp. 2685-2696

31. W Cheng, A Thaeler, X Cheng, F Liu, X Lu, Z Lu, in Proceedings. The Sixth Workshop on Wireless Ad Hoc and Sensor Networks (WWASN2009). Time-synchronization free localization in large scale underwater acoustic sensor networks, (2009)

32. M Granovetter, The strength of weak ties. Am. J. Sociol. 78(6), 1360-1380 (1973)

33. MC Gonzalez, CA Hidalgo, A-L Barabasi, Understanding individual human mobility patterns. Nature 453(7196), 779-782 (2008)

34. A Keränen, J Ott, T Kärkkäinen, in Proceedings. The 2nd International Conference on Simulation Tools and Techniques. The one simulator for $\mathrm{dtn}$ protocol evaluation, (2009), p. 55

35. S Jain, K Fall, R Patra, Routing in a delay tolerant network. ACM, USA 34(4), 145-158 (2004)

36. A Lindgren, A Doria, O Schelén, Probabilistic routing in intermittently connected networks. ACM SIGMOBILE Mobile Comput. Commun. Rev. 7(3), 19-20 (2003)

\section{Submit your manuscript to a SpringerOpen ${ }^{\circ}$ journal and benefit from:}

- Convenient online submission

- Rigorous peer review

- Immediate publication on acceptance

- Open access: articles freely available online

- High visibility within the field

- Retaining the copyright to your article 